\title{
A DEMAND ADJUSTMENT PROCESS
}

by

\author{
Elaine Bennett \\ Michael Maschler \\ William Zame
}

\section{UCLA Department of Economics}

Working Paper No. 724

November 1994 


\title{
A DEMAND ADJUSTMENT PROCESS*
}

\author{
Elaine Bennett \\ Department of Economics \\ Virginia Polytechnic Institute \& State University \\ and \\ Department of Economics \\ University of California at Los Angeles \\ Michael Maschler \\ Department of Mathematics \\ Hebrew University of Jerusalem \\ William R. Zame \\ Department of Economics \\ University of California at Los Angeles
}

November, 1994

*The hospitality of the Center for Rationality and the financial support of the National Science Foundation and the UCLA Academic Senate Committee on Research are gratefully acknowledged. 


\section{Introduction}

Aspiration solutions for cooperative games with side payments were introduced by Cross (1967) (who used the term "supercore"). They have been re-discovered, and different selections from the set of aspirations have been suggested as solutions, by Albers (1974, 1980), Bennett (1983) and Turbay (1977); extensions to games without sidepayments have been made by Bennett and Zame (1988), and to spatial games by Albers (1980), Bennett and Winer (1984) and Sharkey (1992). ${ }^{1}$ Aspirations also arise as solutions of non-cooperative bargaining models. In the context of games with sidepayments, such a model was described and analyzed by Selten (1981), who used the framework of recursive games. In Selten's model, the actions of players are to make, accept and/or reject proposals. Bennett (1988, 1991a) cast Selten's proposal-making model in the framework of extensive form games, and extended the analysis to the context of games without sidepayments. In both cases, aspirations define precisely the outcomes that can be supported in a stationary subgame perfect equilibrium. ${ }^{2}$ An axiomatic characterization of aspirations has been given by Moldovanu and Winter (1994). Aspiration solutions also arise in fair division problems (see Moulin (1990)), in comparative statics analysis of core payoffs (see Engl and Scotchmer (1992)), and as multilateral solutions (see Bennett (1986)). For more detailed bibliography, see Bennett (1991a) and Moldovanu and Winter (1994).

Missing from these analyses is a model of how aspirations arise; the purpose of this paper is to provide such a model, in the context of cooperative games without sidepayments (NTU games, for short). The formalization we give is motivated in part by the Albers and Selten interpretation of aspirations as vectors of demands, and by the behavior of subjects in experimental games, observed by Albers (1987), Albers and Laing (1991) and Laing (1991). Given an NTU game and an (arbitrary) initial vector of demands, we suppose that players make adjustments based on the availability of coalitions

\footnotetext{
${ }^{1}$ Albers and Selten used the term "semi-stable demand vectors;" the aspiration terminology is due to Bennett.

${ }^{2}$ Stationarity is crucial to this result. In its absence, Bennett (1991b) obtains a folk theorem: Every individually rational outcome can be supported in a subgame perfect equilibrium.
} 
that support their demands. We assume only three things about the adjustment process:

(i) only one player adjusts at a time

(ii) a player will increase his demand if some coalition can support the larger demand, given the demands of others

(iii) a player will decrease his demand if no coalition can support his current demand, given the demands of others

Note that we make no assumption about which player will adjust his demand at any particular point in time, or about the size of any of the adjustments. Thus, the adjustment process gives rise to a set-valued dynamical system, of the kind introduced by Maschler and Peleg (1976). It is shown that, starting from an arbitrary vector of demands, and no matter how the process evolves, it always converges; under a mild restriction, it converges to an aspiration. If each player only makes adjustments which are as large as possible, the process ends in an aspiration in a finite number of steps. Moreover, each aspiration is a stable point (in the sense of Lyapunov) for this dynamical system.

Following this Introduction, Section 2 collects basic terminology about NTU games and aspirations. Section 3 describes the adjustment process as a set-valued dynamical system, and establishes its basic properties. Convergence is addressed in Section 4, and stability is addressed in Section 5. 


\section{$2 \quad$ NTU Games and Aspirations}

A game in characteristic function form with non-transferable utility (an NTU game $)$ is a pair $\langle N, V\rangle$, where $N=\{1, \ldots, n\}$ is a non-empty set of players and $V$, the characteristic function, assigns to each non-empty subset $S \subseteq N$ (a coalition) a compact subset $V(S) \subset \mathbb{R}_{+}^{S}$ that contains the origin and is comprehensive (that is, if $x \in V(S)$ and $y \in \mathbb{R}_{+}^{S}$ with $y \leq x$ then $y \in V(S)$ ). We restrict our attention to games for which each of the sets $V(S)$ satisfies the stronger property:

- If $x \in V(S), y \in \mathbb{R}_{+}^{S}$ and $y<x$ then $y \in \operatorname{int} V(S)$, the interior of $\mathrm{V}(\mathrm{S})$ with respect to $\mathbb{R}_{+}^{S}{ }^{3}$ (Equivalently: if $z \in V(S)$, and there is a vector $z^{\prime} \in V(S)$ with $z^{\prime}>z$ then there is a vector $z^{\prime \prime} \in V(S)$ with $z^{\prime \prime}>>z$.)

We say that the sets $V(S)$ are strongly comprehensive if they enjoy this property, and that the game $\langle N, V\rangle$ is strongly comprehensive if each of the sets $V(S)$ is strongly comprehensive. (Note that if $V(S)$ is strongly comprehensive, then the weak and strong Pareto boundaries of $V(S)$ coincide, and are equal to the topological boundary of $V(S)$ with respect to $\mathbb{R}_{+}^{S}$ ) We do not require that the sets $V(S)$ be convex or that the game be superadditive. ${ }^{4}$

If $T$ is a non-empty subset of $S$, we write $x^{T}$ for the restriction of $x$ to $T$ (thinking of vectors in $\mathbb{R}^{S}$ as functions from $S$ to $\mathbb{R}$ ). For $i \in S, x \in \mathbb{R}^{S}$ and $t \in \mathbb{R}$, we write $\left(x_{-i}, t\right)$ for the vector in $\mathbb{R}^{S}$ whose $i$-th coordinate is $t$ and which agrees with $x$ in every coordinate except the $i$-th. For $x, y \in \mathbb{R}^{N}$, we write $|x|=\sum\left|x_{i}\right|$ and $d(x, y)$ for the Euclidean distance from $x$ to $y$.

We interpret a vector in $V(S)$ as a vector of utilities which the coalition $S$ can achieve without the cooperation of players not in $S$. Comprehensiveness of $V(S)$ means that we allow for free disposal of utility. Strong comprehensiveness means that, at each feasible utility vector for a coalition, it is

\footnotetext{
${ }^{3}$ Here and elsewhere we write, for $x, y \in \mathbb{R}^{S}: x \leq y$ if $x_{i} \leq y_{i}$ for each $i \in S ; x<y$ if $x \leq y$ and $x \neq y$; and $x<<y$ if $x_{i}<y_{i}$ for each $i \in S$.

${ }^{4} \mathrm{NTU}$ games are sometimes formalized so that $V(S)$ is a subset of $\mathbb{R}^{N}$, unrestricted in the coordinates not belonging to $S$, but we find the present formalization more convenient for our purposes.
} 
possible for each player whose utility level is strictly positive to improve the utility of all other members of the coalition at the (possible) sacrifice of some of his own utility.

We regard vectors $x \in \mathbb{R}_{+}^{N}$ as vectors of demands, so that $x_{i}$ is player $i$ 's demand. We say that player $i$ 's demand is realizable at $x$ if there is a coalition $S$ containing $i$ such that $x^{S} \in V(S)$. The vector $x$ of demands is realizable if each player's demand is realizable. We say that player $i$ 's demand is maximal at $x$ if there does not exist a coalition $S$ containing $i$ and a vector $y^{S} \in V(S)$ such that $y_{i}^{S}>x_{i}$. The vector $x$ of demands is maximal if each player's demand is maximal. An aspiration is a demand vector that is both realizable and maximal for every player. ${ }^{5}$

\footnotetext{
${ }^{5}$ We follow the terminology of Bennett (1983) and Bennett and Zame (1988); Selten (1981) and others use the term semi-stable demand vectors, rather than aspiration.
} 


\section{The Adjustment Process}

We begin by recalling some notions from Maschler and Peleg (1976). Let $X$ be a complete metric space with distance function $d$. A (set-valued) dynamical system on $X$ is a correspondence $\Phi: X \rightarrow X$ with non-empty values. A trajectory of $\Phi$ is a sequence $x^{0}, x^{1}, \ldots$ such that $x^{t+1} \in \Phi\left(x^{t}\right)$ for each $t$; we say that this trajectory starts at $x^{0}$, and that $x^{0}$ is the initial point of the trajectory. An endpoint of $\Phi$ is a point $x$ such that $\Phi(x)=\{x\}$.

Given a game $\langle N, V\rangle$, we define a dynamic demand-adjustment process in the following way. Set $X=\mathbb{R}_{+}^{N}$, and let $d$ be the usual Euclidean distance function. Define functions $c_{i}: X \rightarrow \mathbb{R}_{+}, c: X \rightarrow \mathbb{R}_{+}^{N}$, and $\lambda_{i}: X \rightarrow \mathbb{R}_{+}^{N}$ by:

$$
\begin{aligned}
c_{i}(x) & =\max _{S \subseteq N, i \in S}\left\{t:\left(x_{-i}, t\right)^{S} \in V(S)\right\} \\
c(x) & =\left(c_{1}(x), \ldots, c_{n}(x)\right) \\
\lambda_{i}(x) & =\left(x_{-i}, c_{i}(x)\right)
\end{aligned}
$$

The number $c_{i}(x)$ is the highest realizable demand that player $i$ could make, given the demands of other players. The vector $\lambda_{i}(x)$ is the vector that results from $x$ if player $i$ changes his demand to $c_{i}(x)$ and all other demands remain unchanged; $c(x)$ is the vector that results from $x$ if all players simultaneously change their demands. When $c_{i}(x)<x_{i}$, player $i$ 's demand is not realizable; when $c_{i}(x)>x_{i}$, player $i$ 's demand is not maximal. Bennett and Zame (1988, Theorem 1) show that the functions $c_{i}, c, \lambda_{i}$ are all continuous. ${ }^{6}$

Define the demand adjustment process $\Phi$ by

$$
\Phi(x)=\left\{s \lambda_{i}(x)+(1-s) x: 0 \leq s \leq 1, i \in N\right\}
$$

When $c(x)=x$, each player's demand is realizable - so no player needs to adjust his demand - and maximal - so no player will be able to adjust his demand; in this case $\Phi(x)=\{x\}$. When $c(x) \neq x, \Phi(x)$ is the set of all vectors obtained when one player adjusts his demand.

As suggested in the Introduction, the intuition behind this adjustment process is quite simple. Suppose the current vector of demands is $x$, and

\footnotetext{
${ }^{6}$ This is a consequence of strong comprehensiveness.
} 
that player $i$ contemplates adjusting his demand. (We assume throughout that players are polite, in the sense that only one player at a time can adjust his demand. It would be easy to allow for the possibility that more than one player might adjust at a given time, and our results would not change in any substantial way.) We suppose that player $i$ will increase his demand if there is some coalition that can support the larger demand, and that player $i$ will decrease his demand if there is no coalition that can support his current demand (given the demands of others). Thus, if player $i$ adjusts his demand, he will necessarily adjust it in the direction of $c_{i}(x)$, so the new demand vector must be a convex combination of $x$ and the vector $\lambda_{i}(x)=\left(x_{-i}, c_{i}(x)\right)$. Taking the union over all players, we see that $\Phi(x)$ is precisely the set of demand vectors that can arise from $x$ when a single player adjusts his demand.

The basic properties of the demand adjustment process are contained in the following simple result.

Proposition $1 \Phi$ is a lower hemi-continuous correspondence with non-empty, closed values. The set of endpoints of $\Phi$ is the set of aspirations.

Proof: Evidently, $x \in \Phi(x)$ for each $x$, so that $\Phi$ has non-empty values. Since $\Phi(x)$ is the union of a finite number of closed line segments, $\Phi$ has closed values. To see that $\Phi$ is lower hemi-continuous, consider a sequence $x^{n} \rightarrow x$, and a vector $y \in \Phi(x)$. Write $y=s \lambda_{i}(x)+(1-s) x$. For each $n$, set $y^{n}=s \lambda_{i}\left(x^{n}\right)+(1-s) x^{n}$. The definition guarantees that $y^{n} \in \Phi\left(x^{n}\right)$ for each $n$, and continuity of $\lambda_{i}$ guarantees that $y^{n} \rightarrow y$. Hence $\Phi$ is lower hemi-continuous, as desired. Finally, note that $x$ is an endpoint of $\Phi$ if and only if $c_{i}(x)=x$ for each $i$; that is, each player's demand is realizable and maximal, so that $x$ is an aspiration. 


\section{Convergence of Trajectories}

In this section, we study convergence of trajectories of the adjustment process $\Phi$. We show first that every trajectory converges. In general, a trajectory need not converge to an endpoint, but we identify a natural family of trajectories (maximal trajectories) which do. In general, convergence of trajectories requires infinitely many steps; we identify a natural family of trajectories (greedy trajectories) which converge in a finite number of steps.

We find it convenient to first construct a Lyapunov function for the dynamical system $\Phi$; that is, a continuous function $L: X \rightarrow \mathbb{R}_{+}$that decreases along trajectories and is 0 precisely at endpoints.

For $x \in X=\mathbb{R}_{+}^{N}$, define two sets

$$
\begin{aligned}
& I(x)=\left\{i: c_{i}(x)>x_{i}\right\} \\
& J(x)=\left\{i: c_{i}(x) \leq x_{i}\right\}
\end{aligned}
$$

Clearly, these sets are disjoint and their union is $N$; either of these sets may be empty. We repeatedly use two simple facts about the sets $I(x), J(x)$ :

- if $x \leq y$ then $I(x) \supseteq I(y)$ and $J(x) \subseteq J(y)$

- if $z \in \Phi(x)$ then $I(x) \supseteq I(z)$ and $J(x) \subseteq J(z)$

The first of these facts is obvious from the definition and strong comprehensiveness of the sets $V(S)$. To see the second fact, assume without loss that $z \neq x$, and recall that only one player adjusts his demand at a time, so there is a unique index $k$ such that $z_{k} \neq x_{k}$. If $z_{k}>x_{k}$, then the first fact guarantees that $I(x) \supseteq I(z)$ and $J(x) \subseteq J(z)$. Suppose therefore that $z_{k}<x_{k}$, so that player $k$ lowers his demand. The definition of $\Phi$ guarantees that $x^{S} \notin V(S)$ for each coalition $S$ containing $k$. Thus, for $j \in J(x)$ and $T$ any coalition containing $j$, either $k \notin T$ in which case $z^{T}=x^{T}$, or $k \in T$, in which case $z^{T} \notin$ int $V(T)$. In either case, $j \in J(z)$, as asserted.

For $x \in X$ and $i \in N$, define

$$
h_{i}(x)=\min \left\{d\left(x^{S}, V(S)\right): \text { all } S, i \in S\right\}
$$


and

$$
L(x)=n \sum_{I(x)}\left[c_{i}(x)-x_{i}\right]+\sum_{J(x)} h_{i}(x)
$$

(We follow the usual convention that the empty sum is 0. )

Proposition 2 The function $L$ satisfies the following properties:

1. $L: X \rightarrow \mathbb{R}_{+}$is continuous

2. $L(x)=0$ if and only if $x$ is an endpoint of $\Phi$ (an aspiration)

3. $L(x) \rightarrow \infty$ as $|x| \rightarrow \infty$

4. if $y \in \Phi(x)$ and $y \neq x$ then $L(x)>L(y)$.

5. if $y \in \Phi(x)$ and $y \geq x$ then $L(x)-L(y) \geq d(x, y)$

Proof: $L(x)$ is non-negative, since it is the sum of non-negative terms. To see that $L$ is continuous, note that $d(x, V(S))=0$ whenever $x \in V(S)$, so that an alternative expression for $L$ is

$$
L(x)=n \sum_{i \in N}\left[c_{i}(x)-x_{i}\right]^{+}+\sum_{i \in N} h_{i}(x)
$$

Thus, $L$ is the sum of continuous functions, and is therefore continuous. To see that $L(x)=0$ precisely when $x$ is an endpoint, note that $L(x)=0$ precisely when all the terms making up $L$ are 0 . Hence $L(x)=0$ precisely when, for each player $i, c_{i}(x) \leq x_{i}$ (that is, $x$ is maximal for player $i$ ) and $h_{i}(x)=0$ (that is, $x$ is realizable for player $i$ ). Hence $L(x)=0$ exactly when $x$ is an aspiration. To see assertion 3 , consider a sequence $\left\{x^{k}\right\}$ such that $\left|x^{k}\right| \rightarrow \infty$. We can partition the entire sequence $\left\{x^{k}\right\}$ into finitely many subsequences $\left\{y^{k}\right\}$, each having the property that $y_{i}^{k} \rightarrow \infty$ for some fixed index $i$. Clearly, then, $h_{i}\left(y^{k}\right) \rightarrow \infty$, whence $L\left(y^{k}\right) \rightarrow \infty$. Since this obtains for each of the subsequences into which we have partitioned the original sequence $\left\{x^{k}\right\}$, we conclude that $L\left(x^{k}\right) \rightarrow \infty$, as desired.

To see that $L$ decreases along trajectories, fix $x \in \mathbb{R}_{+}^{N}$ and $y \in \Phi(x)$ with $y \neq x$. Since only one player adjusts his demand at a time, there is a unique index $k$ such that $y_{k} \neq x_{k}$. There are two cases to consider. 
Case 1: $y<x$. By definition, $y_{k}<x_{k}$ so $c_{k}(x)<x_{k}$. We claim that $I(x)=I(y)$ and that $c_{i}(x)=c_{i}(y)$ for $i \in I(x)=I(y)$. To see this, note that the first fact above shows that $I(x) \subseteq I(y)$. To obtain the remainder of the claim, fix $l \in I(y)$. By definition, $c_{l}(y) \geq y_{l}$, so there is a coalition $S$ containing $l$ for which $y^{S} \in \operatorname{int} V(S)$. If $k \in S$ then $x^{S} \notin V(S)$ (because $\left.c_{k}(x)<x_{k}\right)$; since $y<x$, it follows that $y^{S} \notin$ int $V(S)$, which is a contradiction. Hence $k \notin S$, so that $x^{S}=y^{S} \in$ int $V(S)$ and $c_{l}(x)>x_{l}$. Thus, $l \in I(x)$, so $I(x)=I(y)$. This argument also shows that if $l \in S$ and either $x^{S}$ or $y^{S}$ belong to int $V(S)$, then both $x^{S}$ and $y^{S}$ do, and $k \notin S$, so that $x^{S}=y^{S}$. It follows that $c_{l}(x)=c_{l}(y)$.

Since $I(x)=I(y)$, taking complements implies that $J(x)=J(y)$. For $j \in J(x)=J(y)$, strong comprehensiveness and the fact that $y<x$ imply that $d\left(y^{S}, V(S)\right) \leq d\left(x^{S}, V(S)\right)$ for each coalition $S$, whence $h_{j}(y) \leq h_{j}(x)$. Moreover, $h_{k}(y)<h_{k}(x)$ since $y_{k}<x_{k}$.

Putting all of this together with the definition of $L$, we conclude that $L(y)<L(x)$, as desired. This completes the argument in case 1 .

Case 2: $y>x$. We assert that, in moving from $x$ to $y$, player $k$ 's contribution to $L$ decreases by precisely $n\left(y_{k}-x_{k}\right)$, and that every other player's contribution to $L$ either decreases or else increases by at most $\left(y_{k}-\right.$ $\left.x_{k}\right)$. Since there are $n-1$ players other than player $k$, this will yield the desired inequality: $L(x)-L(y) \geq d(x, y)$.

Because player $k$ is the only player to change his demand, it follows that $c_{k}(x)=c_{k}(y)$. If $y_{k}<c_{k}(x)=c_{k}(y)$ (so that player $k$ does not increase his demand as much as possible), then $c_{k}(x)=c_{k}(y)>y_{k}$, so $k \in I(y)$ and

$$
\left[c_{k}(x)-x_{k}\right]-\left[c_{k}(y)-y_{k}\right]=y_{k}-x_{k}
$$

If $y_{k}=c_{k}(x)=c_{k}(y)$ (so that player $k$ does increase his demand as much as possible), then $k \in J(y)$ but $h_{k}(y)=0$. In either case, we see that player $k$ 's contribution to $L$ decreases by precisely $n\left(y_{k}-x_{k}\right)$, as asserted.

To estimate the change in other player's contributions to $L$, consider first a player $i \in I(y)$. Since $y_{i}=x_{i}$ and $y>x$, it follows that $i \in I(x)$ and $c_{i}(y) \leq c_{i}(x)$, so that player $i$ 's contribution to $L$ does not increase. Finally, consider a player $j \in J(y)$. If $j \in J(x)$, then player $j$ 's contribution to 
$L$ increases by $h_{j}(y)-h_{j}(x)$; if $j \notin J(x)$, then player $j$ 's contribution to $L$ increases by at most $h_{j}(y)$. For each coalition $S$ such that $j \in S$ and $y^{S} \notin$ int $V(S)$, it follows from the triangle inequality that

$$
d\left(y^{S}, V(S)\right) \leq d\left(x^{S}, V(S)\right)+d(x, y)
$$

Noting that $d(x, y)=y_{k}-x_{k}$ and applying the definition of $h_{j}(y)$, we conclude that player $j$ 's contribution to $L$ increases by at most $y_{k}-x_{k}$, as asserted. Combining the change in player $k$ 's contribution with these estimates of the changes in other player's contributions yields $L(y)<L(x)$. This completes the argument in case 2, and with it the proof of Proposition 2.

Following Maschler and Peleg (1976), we say that the trajectory $x^{0}, x^{1}, \ldots$ is $\alpha$-maximal if $\alpha>0$ is a positive real number and

$$
d\left(x^{t+1}, x^{t}\right) \geq \alpha \max _{i}\left|c_{i}(x)-x_{i}\right|
$$

for infinitely many indices $t$. We say that the trajectory $x^{0}, x^{1}, \ldots$ is maximal if it is $\alpha$-maximal for some $\alpha>0$. If we interpret $x_{i}$ as player $i$ 's current demand and $c_{i}(x)$ as player $i$ 's new demand, then $\left|c_{i}(x)-x_{i}\right|$ is the magnitude of the change in player $i$ 's demand, and $\max _{i}\left|c_{i}(x)-x_{i}\right|$ is the maximum magnitude of all these changes. To say that the trajectory is $\alpha$-maximal means therefore that at least the given fraction $\alpha$ of these maximal changes is implemented infinitely often.

We are now in a position to show that all trajectories converge and that maximal trajectories converge to endpoints (which are aspirations).

Theorem 1 Every trajectory converges. Every maximal trajectory converges to an aspiration.

Proof: Let $x^{0}, x^{1}, \ldots$ be a trajectory, so that $x^{t+1} \in \Phi\left(x^{t}\right)$ for each $t$. Convergence of the sequence $\left\{x^{t}\right\}$ is equivalent to convergence of the series $\sum\left[x^{t}-x^{t+1}\right]$. To establish convergence of this series it is convenient to write $U$ for the set of indices $t$ such that $x^{t+1} \geq x^{t}$, and $D$ for the complementary set of indices. We claim that each of the sums

$$
\sum_{U}\left[x^{t}-x^{t+1}\right] \quad \text { and } \quad \sum_{D}\left[x^{t}-x^{t+1}\right]
$$


is convergent - in fact, absolutely convergent. To see this, observe that the first of these sums is composed entirely of non-positive terms, while the second sum is composed entirely of non-negative terms; hence to establish absolute convergence of these sums it suffices to show merely that they are bounded.

Proposition 2 guarantees that $L\left(x^{t}\right)-L\left(x^{t+1}\right) \geq d\left(x^{t}, x^{t+1}\right)$ for $t \in U$, and that $L\left(x^{t}\right)-L\left(x^{t+1}\right) \geq 0$ for every $t$. Since only one player adjusts his demand at a time, $d\left(x^{t}, x^{t+1}\right)=\left|x^{t}-x^{t+1}\right|$ for every $t$. Hence

$$
\begin{aligned}
\left|\sum_{U}\left[x^{t}-x^{t+1}\right]\right| & \leq \sum_{U}\left|x^{t}-x^{t+1}\right| \\
& \leq \sum_{U}\left[L\left(x^{t}\right)-L\left(x^{t+1}\right)\right] \\
& \leq \sum_{t=0}^{\infty}\left[L\left(x^{t}\right)-L\left(x^{t+1}\right)\right]
\end{aligned}
$$

This last series telescopes; since $L$ is non-negative and decreasing along trajectories, it converges to

$$
L\left(x^{\mathbf{0}}\right)-\lim _{t \rightarrow \infty} L\left(x^{t}\right)
$$

Thus, the summation over $U$ is bounded.

To see that the summation over $D$ is bounded, note that the entire summation telescopes; a typical partial sum is

$$
\sum_{t=0}^{T}\left[x^{t}-x^{t+1}\right]=x^{0}-x^{T+1}
$$

Since $L$ decreases along trajectories, $L\left(x^{T+1}\right) \leq L\left(x^{0}\right)$. Since $L$ tends to $\infty$ along unbounded sequences, it follows that the partial sums above remain bounded. The partial sums of the summation over $D$ are differences of these partial sums and the partial sums of the summation over $U$, and are thus bounded.

We conclude that the summations over $U$ and over $D$ are bounded, and hence absolutely convergent. We may then write

$$
\sum_{t=0}^{\infty}\left[x^{t}-x^{t+1}\right]=\sum_{U}\left[x^{t}-x^{t+1}\right]+\sum_{D}\left[x^{t}-x^{t+1}\right]
$$


and conclude that the series $\sum\left[x^{t}-x^{t+1}\right]$ converges, and hence that the sequence $\left\{x^{t}\right\}$ converges, as desired.

Finally, assume that $x^{0}, x^{1}, \ldots$ is a maximal trajectory, and hence is $\alpha$ maximal for some $\alpha>0$. If $x^{t} \rightarrow x$ and $x$ is not an aspiration, then $c_{i}(x) \neq x_{i}$ for some $i$, so

$$
\left|c_{i}(x)-x_{i}\right|>\delta>0
$$

for some $\delta$. Continuity of $c_{i}$ implies that $\left|c_{i}\left(x^{t}\right)-x_{i}^{t}\right| \geq \delta$ for each $t$ sufficiently large, and $\alpha$-maximality implies that $d\left(x^{t+1}, x^{t}\right) \geq \alpha \delta$ for infinitely many $t$. On the other hand, since $x^{t} \rightarrow x$, it follows that $d\left(x^{t}, x^{t+1}\right) \rightarrow 0$, so we have arrived at a contradiction. This completes the proof.

This result shows that our demand adjustment procedure always converges, and converges to an aspiration provided that a fixed fraction of the maximum of demands is satisfied infinitely often. Such convergence, however, could be arbitrarily slow. ${ }^{7}$ In particular, convergence may require an infinite number of steps. However, if each individual adjustment is as large as possible, we can show that convergence occurs in a finite number of steps. To make this precise, let us say that the trajectory $x^{0}, x^{1}, \ldots$ is greedy if for each $t$, either $x^{t}$ is an aspiration or there is a player $k$ such that $x_{k}^{t} \neq x_{k}^{t+1}$ and $x^{t+1}=\lambda_{k}\left(x^{t}\right)$ (equivalently: $x_{k}^{t+1}=c_{k}\left(x^{t}\right)$ ); of course this means that the player who adjusts his demand actually adjusts as much as possible. Note that the set of greedy trajectories forms a proper subset of the set of all trajectories, so we should expect the set of limit points of optimal trajectories to be a proper subset of the set of limit points of all trajectories.

Theorem 2 If the trajectory $x^{0}, x^{1}, \ldots$ is greedy, then $x^{2^{n}-1}$ is an aspiration, and the trajectory is constant from that point on.

Before beginning the proof proper, it is convenient to isolate two lemmas. The first of these represents a purely technical combinatorial portion of the argument. If $A$ is a finite set, a word on $A$ is a finite expression of the form $w=a_{1} a_{2} \ldots a_{M}$, where $a_{m} \in A$ for each $m$; we call $M$ the length of the word

\footnotetext{
${ }^{7}$ If the adjustment at every step is a fixed fraction of the maximum possible at that step, it is easy to show that convergence is at a geometric rate.
} 
$w$. (The empty set is a word of length 0 .) If $w_{1}, w_{2}$ are words, so is the concatenation $w_{1} w_{2}$. We write $W(A)$ for the set of words on $A$. If $B \subseteq A$, we write $W(A, B)$ for the set of words $w \in W(A)$ that contain every element of $B$.

Lemma 1 If $A$ is a non-empty set containing $r \geq 1$ elements and $w \in W(A)$ is a word of length $M \geq 2^{r}$, then there are a proper subset $B \subset A$, an element $a \in A \backslash B$, and words $\gamma \in W(A, B), \beta \in W(B), \zeta \in W(A)$ such that $w=\gamma a \beta a \zeta^{8}$

Proof: We proceed by induction on $r$. If $r=1$, and $w$ is a word of length $M \geq 2^{r}=2$, we take $B=\emptyset$ and $\beta$ and $\zeta$ to be the empty word, and write $w=(a \ldots a) a \beta a \zeta$, where $(a \ldots a)$ has length $M-2$.

Assume therefore that the Lemma is valid for all sets having at most $r-1$ elements; let $A$ be a set with $r$ elements, and let $w \in W(A)$ be a word of length $M \geq 2^{r} \geq 4$. Write $w=\varphi \psi$, where $\varphi$ has length $2^{r-1}$. If some element of $A$ does not occur in $\varphi$, then $\varphi \in W\left(A^{\prime}\right)$ for some proper subset $A^{\prime} \subset A$. Applying our induction hypothesis to $\varphi, A^{\prime}$ allows us to choose a proper subset $B \subset A^{\prime}$, and words $\gamma \in W\left(A^{\prime}, B\right), \beta \in W(B), \zeta \in W\left(A^{\prime}\right)$ such that $\varphi=\gamma a \beta a \zeta$, whence $w=\gamma a \beta a(\zeta \psi)$, which is the desired representation. We may therefore suppose that every element of $A$ occurs in $\varphi$. Since $w$ has length $2^{r}$ and $\varphi$ has length $2^{r-1}$, the word $\psi$ has length at least $2^{r-1}$, so the same argument shows that we may also suppose that every element of $A$ occurs in $\psi$.

Let $b$ be the last letter of $\varphi$, so that $\varphi=\varphi^{\prime} b$ and $w=\varphi^{\prime}(b \psi)$. Since every element of $A$ occurs in $\psi$, we can find words $\beta, \nu$ so that $\psi=\beta b \nu$, and $b$ does not occur in $\beta$. (Simply take $\beta$ to be the word consisting of all letters preceeding the first occurrence of $b$ in $\psi$. If $\psi$ begins with $b$, then $\beta$ is the empty word.) Hence $w=\varphi \psi=\varphi^{\prime} b \beta b \nu$; if we take $B=A \backslash\{b\}$, this is the desired representation.

The second lemma provides some information about the adjustment process; we will also find this information useful in the next section.

\footnotetext{
${ }^{8}$ We allow for the possibility that $B$ is the empty set, in which case $\beta$ will necessarily be the empty word.
} 
Lemma 2 Fix $w \in \mathbb{R}^{N}, z \in \Phi(w)$ and $j \in N$. Then:

(i) If $c_{j}(w) \leq w_{j}$ then $c_{j}(z) \leq z_{j}$. Thus, a player who cannot raise his demand now will never be able to raise his demand.

(ii) If $c_{j}(w)>w_{j}$ then $c_{j}(z) \leq c_{j}(w)$. Thus, a player who can raise his demand now will never be able to raise his demand above the level to which he can raise it now.

Proof: To verify (i), assume without loss that $z \neq w$; let $k$ be the unique player for whom $z_{k} \neq w_{k}$. If $k=j$ the assertion is a tautology, so assume that $k \neq j$. If $k$ raises his demand (in going from $w$ to $z$ ) then $z \geq w$ and $c_{j}(z) \leq c_{j}(w)$ because $c_{j}$ is a decreasing function. On the other hand, if $k$ lowers his demand, then the vector $w$ is not realizable for $k$, so there does not exist a coalition $S$ containing $k$ with $w^{S} \in V(S)$. If $c_{j}(z)>z_{j}$, then there is a coalition $T \subset N$ containing $j$ such that $z^{T} \in \operatorname{int} V(T)$. In view of the previous comment, the coalition $T$ cannot contain player $k$, so that $w^{T}=z^{T}$ and $c_{j}(w)=c_{j}(z)>z_{j}=w_{j}$, which is a contradiction. This yields (i).

The argument for (ii) is similar. Assume without loss that $z \neq w$; let $k$ be the unique player for whom $w_{k} \neq z_{k}$. If $k=j$ the assertion is a tautology, so assume that $k \neq j$. If $k$ raises his demand (in going from $w$ to $z$ ) then $z \geq w$ and $c_{j}(z) \leq c_{j}(w)$ because $c_{j}$ is a decreasing function. On the other hand, if $k$ lowers his demand then the vector $w$ is not realizable for $k$, so there does not exist a coalition $S$ containing $k$ with $w^{S} \in V(S)$. The definition of $c_{j}(z)$ means that there is a coalition $T$ containing $j$ such that $\left(z_{-j}^{T}, c_{i}(z)\right) \in V(T)$. The previous comment means that the coalition $T$ cannot contain player $k$, so that $w^{T}=z^{T}$, whence $\left(w_{-j}^{T}, c_{j}(z)\right) \in V(T)$ so that $c_{j}(z) \leq c_{j}(w)$, which is the desired result. This yields (ii).

With these lemmas in hand, we turn to the proof of Theorem 2.

Proof of Theorem 2: Fix a greedy trajectory $\left\{x^{t}\right\}=\left\{x^{0}, x^{1}, \ldots\right\}$. If $x^{t}$ is an aspiration then no player can adjust his demand, so $x^{t+1}=x^{t}$. In particular, once the trajectory hits an aspiration, it is constant from that point on. Conversely, if $x^{t}$ is not an aspiration, greediness guarantees that $x^{t+1} \neq x^{t}$; let $k(t)$ be the unique player who adjusts his demand at $x^{t}$. 
Suppose that $x^{2^{n}-1}$ is not as aspiration. In the notation of Lemma 1, $w=k(0) k(1) \ldots k\left(2^{n}-1\right)$ is a word in $W(N)$ of length $2^{n}$. Lemma 1 guarantees that there is a coalition $S \subset N$, a player $k \in N \backslash S$, and words $\gamma \in W(N, S), \beta \in W(S), \zeta \in W(N)$ such that $w=\gamma k \beta k \zeta$. Write $p$ for the length of $\gamma$ and $q$ for the length of $\beta$. We may then write $w$ as

$$
w=\gamma k(p) \beta k(p+q+1) \zeta
$$

where $k(p)=k(p+q+1)=k$. We proceed to obtain a contradiction.

We claim that, although the first price adjustment for each player may be either upward or downward, all subsequent adjustments by the same player are downward. To see this, fix a player $i$ and times $t<t^{\prime}$ at which $i$ adjusts his price. There is no loss of generality in assuming that $t, t^{\prime}$ are successive times of price adjustment for player $i$; that is, $i$ does not adjust his price at any time between $t$ and $t^{\prime}$. If $i$ has lowered his price at time $t$, Lemma 2 guarantees that he cannot raise it at time $t^{\prime}$. That is, if $c_{i}\left(x^{t}\right)<x_{i}^{t}$ then $c_{i}\left(x^{t^{\prime}}\right) \leq x_{i}^{t^{\prime}}$. If $i$ has raised his price at time $t$, Lemma 2 guarantees that he cannot raise it to a higher level at time $t^{t}$. That is, if $c_{i}\left(x^{t}\right)>x_{i}^{t}$ then $c_{i}\left(x^{t^{\prime}}\right) \leq c_{i}\left(x^{t}\right)$. Greediness means that $c_{i}\left(x^{t}\right)=x_{i}^{t+1}$, and the assumption that $i$ does not adjust his price between times $t$ and $t^{\prime}$ means that $x_{i}^{t+1}=x_{i}^{t^{\prime}}$. Combining these again yields $c_{i}\left(x^{t^{\prime}}\right) \leq x_{i}^{t^{\prime}}$, which establishes the claim.

Assembling the information now available will yield the contradiction we seek. Recall that $k$ is the player who is adjusting his price at time $p$ and again at time $p+q+1$, so that $k=k(p)=k(p+q+1)$.

1. Consider indices $t$ in the range $p+1 \leq t \leq p+q$. By construction, the word $\beta$ belongs to $W(S)$; that is, $k(t) \in S$ for each $t$ in the given range. In other words, in the range $p+1 \leq t \leq p+q$ only players in $S$ are adjusting their prices. Moreover, since the word $\gamma$ belongs to $W(N, S)$, each of these players has already adjusted their price at least once before. In view of the claim established above, we conclude that

$$
x^{p+1} \geq x^{p+2} \geq \cdots \geq x^{p+q+1}
$$

Hence

$$
c_{k}\left(x^{p+1}\right) \leq c_{k}\left(x^{p+2}\right) \leq \cdots \leq c_{k}\left(x^{p+q+1}\right)
$$


2. Greediness and the definition of the adjustment process yield

$$
c_{k}\left(x^{p}\right)=x_{k}^{p+1}=c_{k}\left(x^{p+1}\right)
$$

3. Combining these two inequalities yields

$$
c_{k}\left(x^{p}\right) \leq c_{k}\left(x^{p+q+1}\right)
$$

and greediness of the adjustment process gives

$$
c_{k}\left(x^{p}\right)=x_{k}^{p+1} \leq x_{k}^{p+q+2}=c_{k}\left(x^{p+q+1}\right)
$$

4. Applying the claim to player $k$ yields

$$
x_{k}^{p+q+2} \leq x_{k}^{p+q+1}
$$

5. By construction, $k \notin S$, so between times $p+1$ and $p+q$ player $k$ is not adjusting his price. Hence

$$
x_{k}^{p+q+1}=x_{k}^{p+1}
$$

Combining the last three inequalities yields

$$
x_{k}^{p+q+2} \geq x_{k}^{p+1}=x_{k}^{p+q+1} \geq x_{k}^{p+q+2}
$$

Recalling that $k=k(p)=k(p+q+1)$, we conclude that

$$
x^{p+q+2}=x^{p+q+1}
$$

But this means that $x^{p+q+1}$ is an aspiration, whence $x^{2^{n}-1}$ is also an aspiration, which is a contradiction. This completes the proof. 


\section{$5 \quad$ Stability}

As we have shown in the preceding sections, aspirations are the endpoints of the dynamical system $\Phi$, and every maximal trajectory converges to an aspiration. The purpose of this section is to show that every aspiration is a stable point of $\Phi$; trajectories beginning sufficiently near an aspiration $x$ remain near $x$ forever. (Formally: for every aspiration $x$ and every $\epsilon>0$, there is a $\delta>0$ such that if $d(y, x)<\delta$, then every trajectory $y=y^{0}, y^{1}, \ldots$ starting at $y$ has the property that $d\left(x, y^{t}\right)<\epsilon$ for all $t$.)

Theorem 3 Every aspiration is a stable point of the dynamical system $\Phi$.

Proof: We first obtain a priori upper and lower bounds on trajectories starting at an arbitrary given point; stability of aspirations will follow easily from these bounds.

Fix an arbitrary point $y \in \mathbb{R}_{+}^{N}$. Renumbering if necessary, we may assume that $I(y)=\{1, \ldots, m\}$ and that $J(y)=\{m+1, \ldots, n\}$, with $0 \leq m \leq n .^{9}$ Set

$$
\bar{y}=\left(c_{1}(y), \ldots, c_{m}(y), y_{m+1}, \ldots y_{n}\right)
$$

and

$$
\underline{y}=\min \{y, c(\bar{y})\}
$$

We claim that every trajectory $y=y^{0}, y^{1}, \ldots$ beginning at $y$ remains forever between $\underline{y}$ and $\bar{y}$; that is,

$$
\underline{y} \leq y^{t} \leq \bar{y} \text { for each } t
$$

To establish this claim we proceed inductively. It is clear from the definitions that $y \leq y=y^{0} \leq \bar{y}$. Now fix an index $t \geq 0$ and assume that $\underline{y} \leq y^{t} \leq \bar{y}$; we must show that $\underline{y} \leq y^{t+1} \leq \bar{y}$. Let $k$ be the unique player who alters his demand at time $t$, and consider two cases, according to whether $k$ raises or lowers his demand.

\footnotetext{
${ }^{9}$ If $m=0$ then $I(y)$ is empty; if $m=n$ then $J(y)$ is empty.
} 
(1) If $k$ raises his demand at time $t$, Lemma 2 guarantees that he was able to raise it at time 0 (so that $k \in I(y)$ ), and cannot, at time $t$, raise his demand above the level to which he was able to raise it at time 0 . Hence

$$
y_{k}^{t} \leq y_{k}^{t+1} \leq c_{k}\left(y^{t}\right) \leq c_{k}(y)=\bar{y}_{k}
$$

Since $k$ is the only player who alters his demand and $\underline{y} \leq y^{t} \leq \bar{y}$, it follows that

$$
\underline{y} \leq y^{t} \leq y^{t+1} \leq \bar{y}
$$

(2) If $k$ lowers his demand at time $t$, then $y_{k}^{t+1} \leq y_{k}^{t} \leq \bar{y}_{k}$. Moreover, $c_{k}\left(y^{t}\right) \geq c_{k}(\bar{y}) \geq \underline{y}_{k}$ (because $c_{k}$ is a decreasing function). Combining these inequalities, and recalling that $k$ is the only player who alters his demand yields

$$
\underline{y} \leq y^{t+1} \leq y^{t} \leq \bar{y}
$$

We conclude in either case that, if $y^{t}$ satisfies the desired bounds, then $y^{t+1}$ does so also. It follows by induction that the entire trajectory satisfies the desired bounds.

Given these bounds, stability of each aspiration follows easily. Fix an aspiration $x$ and an $\epsilon>0$. Each of the functions $c_{i}$ is continuous, and taking the minimum of two vectors is a continuous function. Moreover, since $x$ is an aspiration, $c_{i}(x)=x_{i}$ for each $i$. Hence, we can find a $\delta>0$ sufficiently small that if $d(y, x)<\delta$ then $d(\bar{y}, x)<\epsilon$ and $d(\underline{y}, x)<\epsilon$. If $y=y^{0}, y^{1}, \ldots$ is any trajectory starting at $y$, then $y \leq y^{t} \leq \bar{y}$ for each $t \geq 1$. Thus, if $d(y, x)<\delta<\epsilon 0$, then $d\left(y^{t}, x\right)<\epsilon$ for each $t \geq$, as required. 


\section{References}

1. W. Albers (1974), "Zwei Losungskonzepte fur Kooperative Mehrpersonspiele, die auf Anspruchsnivaus der Spieler Basieren," OR-Verfahren, 21, 1-13.

2. W. Albers (1980), "Core- and Kernel-variants Based on Imputations and Demand Profiles," in O. Moeschlin and D. Palaschke, eds., Game Theory and Related Topics, North Holland, Amsterdam.

3. W. Albers (1987), "Aspirations and Aspiration Adjustment in Location Games," Working Paper, Institute for Mathematical Economics Research, University of Bielefeld.

4. W. Albers and J. D. Laing (1991), "Prominence, Competition, Learning, and the Generation of Offers in Computer-Aided Experimental Spatial Games," in R. Selten, ed., Game Equilibrium Models, III: Strategic Bargaining, Springer-Verlag, Berlin.

5. E. Bennett (1983), "The Aspiration Approach to Predicting Coalition Formation and Payoff Distribution in Sidepayment Games," International Journal of Game Theory, 12, 1-28.

6. E. Bennett (1986), "Multilateral Bargaining Problems," Working Paper \#594, University of California, Los Angeles.

7. E. Bennett (1991a), "Three Approaches to Bargaining in NTU Games," in R. Selten, ed., Game Equilibrium Models, III: Strategic Bargaining, Springer-Verlag, Berlin.

8. E. Bennett (1991b), "A Folk Theorem for the Proposal Making Model," in R. Selten, ed., Strategic Bargaining, Springer-Verlag, Berlin.

9. E. Bennett and M. Winer (1983), "The Bargaining Equilibrium for Spatial Committee Decision Problems," unpublished.

10. E. Bennett and M. Wooders (1979), "Income Distribution and Firm Formation," Journal of Comparative Economics, 3, 304-317. 
11. E. Bennett and W. Zame (1988), "Bargaining in Cooperative Games," International Journal of Game Theory, 17, 279-300.

12. J. Cross (1967), "Some Theoretical Characteristics of Economic and Political Coalitions," Journal of Conflict Resolution, 11, 184-195.

13. G. Engl and S. Scotchmer (1992), "The Core and the Hedonic Core: Equivalence and Comparative Statics," Working Paper, University of California, Berkeley.

14. J. D. Laing (1991), "A Non-Cooperative Model of Bargaining in Simple Spatial Games," in R. Selten, ed., Game Equilibrium Models, III: Strategic Bargaining, Springer-Verlag, Berlin.

15. M. Maschler and B. Peleg (1976), "Stable Sets and Stable Points of Set-Valued Dynamical Systems with Applications to Game Theory," SIAM Journal of Control and Optimization, 14, 193-213.

16. B. Moldovanu and E. Winter (1992), "Core Implementation and Increasing Returns to Scale for Cooperation," Working Paper, Hebrew University of Jerusalem.

17. B. Moldovanu and E. Winter (1994), "Consistent Demands for Coalition Formation," in N. Megiddo, ed., Essays in Game Theory in Honor of Michael Maschler, Springer-Verlag, New York.

18. H. Moulin (1990), "Cores and Large Cores when Population Varies," International Journal of Game Theory, 19, 219-252.

19. R. Selten (1981), "A Non-Cooperative Model of Characteristic Function Bargaining," in V. Bohm and H. H. Nachtkamp, eds., Essays in Game Theory and Mathematical Economics in Honor of Oskar Morgenstern, Bibliographisches Institut Mannheim, Wien-Zurich.

20. W. Sharkey (1993), "A Characterization of Some Aspiration Solutions with an Application to Spatial Games," Working Paper, BellCore.

21. G. Turbay (1977), On Value Theories for N-person Cooperative Games, Ph.D. Dissertation, Rice University. 